\title{
What price statistical independence? How Einstein missed the photon ${ }^{1}$
}

\section{Simon Saunders}

\begin{abstract}
Einstein's celebrated 1905 argument for light quanta was phrased in terms of the concept of 'statistical independence'- wrongly. It was in fact based on the notion of local, non-interacting entities, and as such was neutral on the question of statistical independence. A specific kind of statistical dependence had already been introduced by Gibbs, in connection with the Gibbs paradox, but went unrecognised by Einstein. A modification of Einstein's argument favours the latter. This kind of statistical dependence, as eventually attributed by Einstein to Bose, although expressed, in quantum mechanics, by the (anti)symmetrisation of the wave-function, does not require 'genuine' entanglement. This chapter demystifies quantum indistinguishability; its novel features derive from the finiteness of the state-space in quantum mechanics, not indistinguishability.
\end{abstract}

\section{\$1 Overview}

According to the physicist and historian Abraham Pais, the photon was the first particle to be predicted theoretically. It was also, he noted, met with scepticism on an epic scale: '[i]ts assimilation came after a struggle more intense and prolonged than for any other particle ever postulated'. ${ }^{2}$ Yet it was also, in a certain sense, derived from empirical data - specifically, derived by Einstein from the Wien limit of Planck's black-body spectral distribution, taken as a phenomenological given (it was by then accurately confirmed at high frequencies and low temperatures). The derivation was what John Norton has called Einstein's 'miraculous argument', contained in the most revolutionary of all of Einstein's great papers of $1905 .^{3}$

If it was inferred from the data, how was it opposed? The answer in part is that it was incomplete: whatever light was really made of, it could not only be Einstein's light quanta, because if it were, it would contravene the Planck distribution in the low frequency hightemperature regime (the Rayleigh-Jeans regime, also by then accurately confirmed). What was the nature of the struggle, that was so intense and prolonged? The answer in part was the difficulty of reconciling a particulate structure to radiation with Maxwell's equations (or any wave equation, needed to explain interference effects), but another part was down to Einstein's insistence on statistical independence. It was Einstein's struggle; and it was only concluded (and then only temporarily) with his quantum theory of the ideal monatomic gas, published in three instalments in the period 1924-5.

To see the nature of the difficulty, consider that radiation in thermal equilibrium behaves as a gas of indistinguishable particles, photons, satisfying the Bose-Einstein statistics; and that the latter, as Einstein admitted in his second paper of his gas trilogy, implies that particles are not statistically independent of each other; yet Einstein's 'miraculous argument' of 1905 had shown that thermal radiation, in the Wien regime, is made of statistically independent particles. Contradiction.

\footnotetext{
${ }^{1}$ To appear in Shenker and Hemmo (2020), in a volume dedicated to the memory of Itamar Pitowski.

2 Pais (1982 p.361).

${ }^{3}$ Norton (2006).
} 
The obvious answer is to suppose that in the Wien regime photons become statistically independent of each other; and it is true that in this regime they satisfy Maxwell-Boltzmann statistics. However, the latter is not the signature of statistical independence: classical indistinguishable particles, in Gibbs sense, also obey Maxwell-Boltzmann statistics, but they are not statistically independent in the requisite sense.

The better answer is that Einstein did not show that radiation, in the Wien regime, is composed of statistically independent particles. His argument showed that in the Wien regime thermal radiation is made of non-interacting, localised entities; it left open the question of whether they were indistinguishable, in Gibbs' sense, or statistically independent, in Einstein's. However, as we shall see, a slightly different kind of fluctuation than the one Einstein considered resolves the under-determination in favour of the Gibbs concept - for roughly the reasons Gibbs himself advocated it, namely, as a solution to what is now called the Gibbs paradox.

Had Einstein in 1905 understood this point, the history of the development of quantum theory would have been significantly different, for it would have been a relatively short step to Einstein's quantum gas theory. It is therefore something of an embarrassment that Gibbs' concept of indistinguishability, introduced in terms of the notion of 'generic phase' (as opposed to 'specific phase', as had hitherto been implicitly assumed), was to be found in his Elementary Principles in Statistical Mechanics, published in 1902, and indeed already published in German translation in 1904.

It seems that Einstein knew nothing of Gibbs' work at this time, but we know that he had read at least parts of the Principles by 1910 (and that it impressed him greatly) ${ }^{4}$; however, we also know that he was sceptical of Planck's claims, in the period 1912-1918, on the extensivity of the entropy function of a material gas, that cited $\mathrm{Gibbs}^{5}$ (the failure of extensivity is one formulation of the Gibbs paradox). When Einstein embraced essentially the same solution to the extensivity puzzle in the second of his papers on gas theory, it was in the name of what he called 'Bose's statistical approach', not Gibbs'.

Bose himself, by his own admission, was unaware that his approach had differed from the usual one - and, indeed, his procedure so obscured the underlying statistical assumptions that Einstein, when he took it over in his first paper on gas theory, did not realise (or at any rate did not mention) that it implied the failure of statistical independence. In his second paper, published at the beginning of 1925, Einstein presented the expression for the entropy in a different form, from which this failure was 'easily recognised'; but he still attributed the approach to Bose. That expression for the entropy, I am claiming, is the natural transposition of Gibbs' notion of generic phase to the case where the one-particle state space has a finite number of states (when it is divided into 'elementary cells', of size $h^{3}$ ) - the central assumption of the 'old' quantum theory.

No more did Schrödinger identify Gibbs as the progenitor of 'Bose's approach', although he came close. He had publicly criticised Planck's arguments for an extensive entropy, that drew on Gibbs', and now found fault with Einstein's new gas theory precisely on the point that, following Bose's method, particles could not be statistically independent of each other. Einstein, by then, was prepared to grant that it was for nature to decide; not so Schrödinger, who went on (in a paper immediately preceding the first of his historic papers on wave mechanics) to propose a new quantisation method for the quantum gas that maintained

\footnotetext{
${ }^{4}$ As recounted by Pais (1982 p.55).

${ }^{5}$ From correspondence with Ehrenfest in 1918, quoted in Darrigol (1991 p.288-89).
} 
statistical independance. Neither Einstein nor Schrödinger mentioned Gibbs by name, or the concept of generic phase. The following year, in 1926, Dirac laid down the definitive treatment of particle indistinguishability in quantum mechanics, again with no reference to Gibbs (or to Schrödinger on gas theory) - and few physicists have looked back to its precursors since. Historians, meanwhile, seem to have taken Einstein, Schrödinger, and Dirac rather too much at face value; since none of them mentioned Gibbs or the generic phase, the equivalence of the latter with 'Bose's approach' has not been recognised, or even seriously entertained.

There are other reasons why the equivalence was not recognised. One is because BoseEinstein statistics is directly related to the symmetrisation of the state in quantum mechanics (Dirac cited Bose and Einstein). But the latter, by wide consensus, is thought to involve entanglement, a quintessentially quantum concept. That it is in fact entanglement of a particularly trivial kind -- that does not, for example, support the violation of any Bell inequality - has only recently been shown. Another reason the equivalence has not been recognized comes from the classical side, where it is thought that the concept of particle indistinguishability cannot apply to classical mechanics 'because classical particles can always be distinguished by their trajectories'. That phrase, or one like it, recurs again and again in the subsequent literature. ${ }^{6}$ But it meets the counter-argument that quantum particles can also have trajectories -- either approximately, for sufficiently massive and weakly-interacting particles in well-localised states, or exactly, in the case of non-interacting particles, taking 'trajectories' as orbits of orthogonal one-particle states. In neither case do the particles cease to be indistinguishable, in the sense that their total state remains symmetrised. ${ }^{7}$ We shall see a simple example in the concluding section.

It appears that the struggle initiated by Einstein with the photon concept is so intense, and so prolonged, that it continues unabated to this day. I can offer up nothing quite as remarkable as Itamar Pitowsky's discovery, in George Boole's 'impossibilities of experience', of Bell's inequalities. His beautiful theory of correlation polytopes has long been a source of inspiration to me, as has his independence of thought and temperament. But it is my hope that he would have found pleasing the discovery in Gibbs' paradox of the kind of failure of statistical independence on which quantum indistinguishability is based -- as within the realm of possible experience. Jon Dorling, in his acute historical and theoretical observations, was a similar inspiration, and himself wrote on my topic, arguing that in the limit of the Wein regime Einstein's argument showed light quanta were truly point-like. Their friendship and support has always meant much to me. This essay is dedicated to Itamar and to Jon.

\section{§2. The Gibbs Paradox and 'mutual independence'}

Boltzmann's equation

$$
S=k \ln W+\text { const }
$$

was first written down in this notation by Planck, but it was introduced by Boltzmann in his 1877 memoire, often accounted his most important contribution to statistical mechanics. It was derived anew by Einstein in 1905 on the basis of what he called 'Boltzmann's principle',

${ }^{6}$ See, for example, Epstein (1936a p.557), Darrigol (1986 p.205-9), Bach (1997 p.8), Dieks (2014). (Bach's defence of the concept of classical particle indistinguishability extends only to the notion of probability distributions that give no information on individual trajectories.)

\footnotetext{
${ }^{7}$ Saunders (2006 p.199-200, 207).
} 
namely the principle that the entropy of a system is a function of the probability $W$ of its instantaneous state. His argument was as follows: ${ }^{8}$

If it makes sense to talk about the probability of a state of his system, and if, further, each entropy increase can be conceived as a transition to a more probable state, then the entropy $S_{A}$ of a system $A$ is a function of the probability $W_{A}$ of its instantaneous state. Therefore, if we have two systems $S_{A}$ and $S_{B}$ that do not interact with each other, we can put

$$
S_{A}=\varphi_{A}\left(W_{A}\right), \quad S_{B}=\varphi_{B}\left(W_{B}\right) .
$$

If these two systems are viewed as a single system of entropy $S$ and probability $W$, we have

$$
S=S_{A}+S_{B}=\varphi(W)
$$

and

$$
W=W_{A} \cdot W_{B} .
$$

This last relation tells us that the states of the two systems are mutually independent events.

From these equations it follows that

$$
\varphi\left(W_{A} \cdot W_{B}\right)=\varphi_{A}\left(W_{A}\right), \varphi_{B}\left(W_{B}\right),
$$

and from this we get, finally,

$$
\begin{aligned}
& \varphi_{A}\left(W_{A}\right)=C \ln \left(W_{A}\right)+\text { const } \\
& \varphi_{A}\left(W_{A}\right)=C \ln \left(W_{A}\right)+\text { const } \\
& \varphi(W)=C \ln (W)+\text { const. }
\end{aligned}
$$

The quantity $\mathrm{C}$ is thus a universal constant; it follows from the kinetic theory of gases that its value is $R / N$ [Boltzmann's constant $k$, in Planck's notation].

The argument has power and beauty, but we must look to its premises. The left-hand equality of (1) is nowadays called the additivity of the entropy. In thermodynamics, it applies to noninteracting systems $A$ and $B$ each in thermal equilibrium (not necessarily with each other), but Einstein went on to use it to broader effect. Let there be $z$ one-particle states, all equiprobable, so that the probability that a particle will be found in a given state is $1 / z$, and suppose there are $n$ non-interacting particles. They will be 'mutually independent' in Einstein's sense if the probability that they will all be found in that state is $1 / z^{n}$. The joint probability, in other words, should factorise, as the product of probabilities for individual particles considered separately. In this sense, the probability of a set of outcomes on throwing of $n$ dice should be the same, whether the dice are thrown sequentially or all at once, so long as they are thrown independently and do not influence each other.

If thermodynamic probabilities are to factorise in this way when particles are non-interacting, and if they are to connect in a simple way with the available phase space volume, ${ }^{9}$ then the volume measure on the many-particle phase space had better factorise; and that in turn requires that the phase space itself factorises, i.e. that it is the Cartesian product of 1-particle phasespaces $\gamma$ :

\footnotetext{
${ }^{8}$ Einstein (1905 p.94-95); I follow Einstein's notation, save for replacing ' 1 ' and ' 2 ' by ' $A$ ' and ' $B$ '.

${ }^{9}$ I shall use the terms 'probability' and 'phase space volume' more or less interchangeably, and lazily use the same symbol $V$ for both spatial volume and volume of $\gamma$; the context should make clear which is intended.
} 


$$
\Gamma=\gamma \times \ldots \times \gamma
$$

with volume measure:

$$
W_{V}=V^{n}
$$

The dependence of the entropy of $n$ particles on the spatial volume $V$ is then of the form

$$
S_{V}=k \ln V^{n} .
$$

There is the inevitable attendant failure of extensivity: $S_{V}$ as given by (3) does not scale with the extensive quantities, $n$ and $V$. Double the latter and the entropy is not only doubled, but picks up an additional term $2 n k \ln 2$. Of course it is true that extensivity is something of an approximation, as the molecules making up real material gases are at least weakly interacting and there may be boundary effects, but it is an extremely good approximation for thermal radiation, so long as the temperature is not too high, and it follows, or should follow, for an ideal material gas as well.

In terms of the set-up of the Gibbs paradox, suppose two samples of the same ideal gas are confined to volume $V_{A}$ ( $n_{A}$ particles) and $V_{B}$ ( $n_{B}$ particles), at equal pressure and temperature. The initial total entropy is then (from additivity):

$$
S_{A+B}=S_{A}+S_{B}=k \ln V_{A}^{n_{A}}+k \ln V_{B}^{n_{B}} .
$$

Let the two volumes be contiguous and let the two gases subsequently diffuse into each other. Then the final entropy will be:

$$
S_{A \cup B}=k \ln \left(V_{A}+V_{B}\right)^{\left(n_{A}+n_{B}\right)} .
$$

The result is an increase in entropy; in the simplest case $V_{A}=V_{B}$ the difference between (4) and (5) is $\left(n_{A}+n_{B}\right) k \ln 2$. It is the same term spoiling extensivity that we encountered before, where now it reflects the two possibilities for each particle after the partition is removed, whether located in $A$ or in $B$, where before there was only one. This is the form of the Gibbs paradox that we shall be concerned with, where there is no exchange of particles with outside systems, but only between particles in the two volumes $V_{A}$ and $V_{B}$.

In order to obtain no entropy of mixing, and thereby an extensive entropy function, Gibbs proposed to use the quotient of $\Gamma$ as given by (2) under the permutation group $\Pi$ instead, ${ }^{10}$ to use the space of what he called 'generic phases' (now usually called 'reduced phase space'):

$$
\tilde{\Gamma}=\gamma \times \ldots \times \gamma / \Pi \text {. }
$$

It is the space arrived at by identification of points in $\Gamma$ that are related by a permutation of particles (a permutation with respect to which particle has which position and momentum; a permutation of sequence-position in the Cartesian product). The phase space (2) was called by Gibbs the space of 'specific phases'. As Gibbs' remarked, the reduced phase space has a considerably more complicated topology, but the volume measure has a simple expression:

$$
\widetilde{W}_{V}=\frac{V^{n}}{n !} \text {. }
$$

The latter does not factorise, and particles described in this way fail Einstein's test of statistical independence. For suppose particles $n_{A}$ are statistically independent from particles $n_{B}$, both occupying the same region $V$. Then the probability of the instantaneous state of the composite

\footnotetext{
${ }^{10}$ Gibbs did not of course use the concept 'group' and gave no independent motivation for the generic phase other than that it gave no entropy of mixing. It was seen as ad hoc by Lorentz and Ehrenfest; see Darrigol (1991), Saunders (2020) for further discussion.
} 
$\widetilde{W}_{A \cup B}$ should be the product of the probabilities of the state of each, $\widetilde{W}_{A} \cdot \widetilde{W}_{B}$. However, by inspection:

$$
\widetilde{W}_{A \cup B}=\frac{V^{\left(n_{A}+n_{B}\right)}}{\left(n_{A}+n_{B}\right) !} \approx \frac{V^{n_{A}}}{n_{A} !} \cdot \frac{V^{n_{B}}}{n_{B} !}=\widetilde{W}_{A} \cdot \widetilde{W}_{B}
$$

and the probability does not factorise. Contrast the specific probabilities, that do:

$$
W_{A \cup B}=V^{\left(n_{A}+n_{B}\right)}=V^{n_{A}} \cdot V^{n_{B}}=W_{A} \cdot W_{B} .
$$

Returning to the generic phase, our argument used ' $A$ ' and ' $B$ ' as names for the two collections of particles, dispersed through the same volume, when they are supposed to be indistinguishable; is this move legitimate? But surely it is if 'indistinguishable' is merely to renounce factor position in the Cartesian product Eq.(3) - what I shall call the 'realist reading' of the Gibbs' generic phase. The $n$-tuple of pairs in a definite sequence:

$$
\left\langle\left\langle q_{a}, p_{a}\right\rangle,\left\langle q_{b}, p_{b}\right\rangle, \ldots,\left\langle q_{d}, p_{d}\right\rangle\right\rangle \in \Gamma
$$

where $\left\langle q_{a}, p_{a}\right\rangle \in \gamma$, etc., is a specific phase (specific phase space point). The identification of all such points related by a permutation of entries in sequence position, is to pass to a space the points of which are represented by unordered collections:

$$
\left\{\left\langle q_{a}, p_{a}\right\rangle,\left\langle q_{b}, p_{b}\right\rangle, \ldots,\left\langle q_{d}, p_{d}\right\rangle\right\} \in \tilde{\Gamma} .
$$

There is no longer a sequence position to pick out a particle, independent of its position and momentum, but the latter can be specified as such directly, and thereby the particle that has that position and momentum, supposing there are no repetitions. ${ }^{11}$ The identification of points in $\Gamma$ related by permutations does not mean that the resulting space $\tilde{\Gamma}$ does not have points. Nor does it mean that we must interpret those points as an equivalence class of specific phases (sequences); but as Gibbs remarked, the latter is useful from the point of doing analysis on manifolds. ${ }^{12}$

There is nevertheless a fundamental distinction between this way of specifying the partition of the gas of $n$ particles into two collections, each scattered throughout the same spatial volume, and specifying the partition in terms of disjoint volumes instead (and indeed disjoint regions of the one-particle phase space, regions in both momentum space and position space). Specify the sub-systems $A$ and $B$ in the latter way, and we are back to the Gibbs set-up. The generic probability prior to mixing now does factorise: ${ }^{13}$

$$
\widetilde{W}_{A+B}=\widetilde{W}_{A} \cdot \widetilde{W}_{B}=\frac{V^{n_{A}}}{n_{A} !} \cdot \frac{V^{n_{B}}}{n_{B} !} .
$$

\footnotetext{
11 The assumption is innocent in a continuum theory of point-like particles, or for extended but impenetrable molecules, but for a finite state space it is the difference between fermions (no repetitions) and bosons (repetitions).

${ }^{12}$ Gibbs (1902 p.188). Whether my realist reading of the generic phase should be attributed to Gibbs is a delicate question, for Gibbs writings were so brief. For further discussion, see my (2020).

${ }^{13}$ If this is in doubt, consider the analogous question using a discrete state-space, and apply Eq.(16) to find the number of ways of distributing $n_{A}$ indistinguishable particles over $z_{A}$ states, and likewise for $n_{B}$ particles over $z_{B}$ disjoint states; the product of the two numbers is the same as the number of ways of distributing $n=n_{A}+n_{B}$ indistinguishable particles over $z=z_{A}+z_{B}$ states, under the constraint that $n_{A}$ are in $V_{A}$ and $n_{B}$ are in $V_{B}$ (with no regard to which is in which).
} 
If the two gases are allowed to mix, they are no longer statistically independent of each other, as we have seen, and the joint probability is given by the left-hand side of Eq.(7). Using the Sterling approximation:

$$
\ln n ! \approx n \ln n-n
$$

and bearing in mind that since the two gases prior to mixing had the same temperature and pressure, their densities are equal as well, $n_{A} / V_{A}=n_{b} / V_{B}$, it follows after a short calculation:

$$
\widetilde{W}_{A+B} \approx \widetilde{W}_{A \cup B} \text {. }
$$

The reason the equality is only approximate in (8) is because after the gases mix the states available include those in which numbers of particles different from $n_{A}$ and $n_{B}$ may be found in the regions $A$ and $B$ respectively (but not of course by virtue of which particles are found in each of the two regions). After mixing, the available number of microstates is thus increased, but by a number that can be neglected in the Sterling approximation.

The lesson of the Gibbs paradox is that indistinguishable particles, in Gibbs' sense, even if non-interacting, are not statistically independent in Einstein's sense. To put the matter vividly: if there are $n_{A}$ particles alone, initially confined to volume $V_{A}$, expanding into volume $V$, the entropy increases. If there are $n_{B}$ particles alone, initially confined to volume $V_{B}$, with the same density, expanding into volume $V$, the entropy increases. Yet let $n_{A}, n_{B}$ particles be present in each of the two volumes, of the same density, and allowed to expand into volume $V$, then there is no entropy increase, even though the particles are non-interacting, and even though the trajectory of every particle is the same as if all the others were not there. The entropy of the joint system is not the sum of the entropies of the two subsystems, and the entropy change of the joint system is not the sum of the entropy changes of the two subsystems. Einstein's criterion fails for both, and with it mutual independence.

\section{§3. Einstein's 'miraculous argument'}

Consider now Einstein's celebrated argument from the Wien black-body distribution formula. This claimed to show that insofar as that equation is obeyed, light behaves as if made of elements that were 'mutually independent' and, moreover, that were 'moveable points'.

First 'mutually independent'. We must take Einstein to mean what he meant earlier in the same paper, in the argument for the universality of the entropy as a function of $W$ just reviewed. Mutual independence, then, means the factorising of probabilities. Is it the same as the condition that the particles are non-interacting? It has been interpreted in this way, ${ }^{14}$ but that would be a mistake: even if Einstein did ultimately believe that statistical correlations must be explained by some dynamical coupling, he consistently used the phrase 'mutual independence' and later 'statistical independence' (and not 'non-interacting').

Given all this, and given that the Wien limit to the radiation law just is the limit in which Gibbs' measure for the generic phase is valid, there is the obvious difficulty: the probabilities as defined by Gibbs' measure do not factorise, as we have just seen. How then, working from the Wien regime, could Einstein have concluded the probabilities factorise, and hence that light quanta were mutually independent of each other? Indeed, on a careful reading, this was not exactly his claim, nor an accurate reading of his argument. He actually showed that if thermal

\footnotetext{
${ }^{14}$ Most recently by Norton (2006), according to whom 'independence is just the simplest case of no intermolecular forces' (p. 93).
} 
radiation consisted of mutually independent and localised entities, then its spectral distribution would be the Wien distribution. From this we infer that thermal radiation, in the Wien limit, behaves as if made of mutually independent and localised entities, not that it $i$ made of such entities.

Einstein himself, in his summary conclusion, put the onus entirely on mutual independence:

Monochromatic radiation of low density (within the range of validity of Wien's radiation formula) behaves thermodynamically as if it consisted of mutually independent energy quanta of magnitude $h v$. (p.97)

The omission of locality may have been no more than a slip; does he commit the fallacy of affirming the consequent as well? He treads a fine line: light behaves as if it is so constituted, granted; but it so behaves if it is constituted in other ways as well - in particular, if the energy quanta are non-interacting and localised but lack statistical independence, in the very particular way that they are correlated when described by the generic phase.

We clearly need to see the details. From the first law of thermodynamics:

$$
d U=T d S+p d V
$$

it follows:

$$
\left.\frac{d S}{d U}\right|_{V=\text { const }}=\frac{1}{T}
$$

Einstein worked directly with the spectral entropy density $\varphi$ of black-body radiation (the entropy per unit frequency range and volume), as a function of the spectral energy density $\rho$ (the energy per unit frequency and volume), to derive what he called 'the law of black bodies':

$$
\frac{\partial \varphi}{\partial \rho}=\frac{1}{T} .
$$

The Wien spectral distribution law, as first proposed by Wilhelm Wien in 1896, was

$$
\rho=\alpha v^{3} e^{-\beta v / T}
$$

where $\alpha$ and $\beta$ were new and unknown constants. It was well-confirmed by experiment for $v / T \gg 1$, as Einstein noted. Using (10) to write the inverse temperature as a function of $\rho$ and the frequency, and inserting in (9), Einstein obtained a differential equation for $\varphi$. It could readily be integrated to obtain:

$$
\varphi=-\frac{\rho}{\beta v}\left(\ln \frac{\rho}{\alpha v^{3}}-1\right) .
$$

Planck had found this equation in 1898, likewise working from the Wien distribution (and he had used the same method again in 1900, to obtain an equation for the entropy as a function of $\rho$, working from the Planck distribution). Einstein's originality lay in what came next.

Let $S_{0}=V_{0} \varphi$ be the entropy of the radiation in volume $V_{0}$ per unit frequency range and let $E$ be the energy $V_{0} \rho$. Multiplying both sides of (11) by $V_{0}$, it follows, writing $\rho$ in terms of $E$ and $V_{0}$ :

$$
S_{0}=-\frac{E}{\beta v}\left(\ln \frac{E}{\alpha v^{3} V_{0}}-1\right) .
$$

Suppose now that this volume of radiation (in the given frequency range) undergoes a 
spontaneous fluctuation, and is contained instead in the sub-volume $V$; then it will have the entropy:

$$
S=-\frac{E}{\beta v}\left(\ln \frac{E}{\alpha v^{3} V}-1\right) .
$$

The entropy change is therefore:

$$
S-S_{0}=\frac{E}{\beta v} \ln \frac{V}{V_{0}}
$$

or more suggestively:

$$
S-S_{0}=k \ln \left(\frac{V}{V_{0}}\right)^{\frac{E}{k \beta v}} .
$$

It is the same volume-dependence of the entropy function as for an ideal gas of $n=E / k \beta v$ particles; in Planck's notation, for $n=E / h v$ particles; in Einstein's words, as for a gas of $n$ independently movable points.

Here is Einstein's argument in full: ${ }^{15}$

Let us consider a part of the volume $V_{0}$ of magnitude $V$ and let all $n$ moveable points be transferred into the volume $V$ without any other change in the system. It is obvious that this state has a different value of entropy $(S)$, and we now wish to determine the entropy difference with the aid of Boltzmann's principle.

We ask: How great is the probability of the last-mentioned state relative to the original one? Or: How great is the probability that at a randomly chosen instant of time all $n$ independently movable points in a given volume $V_{0}$ will be contained (by chance) in volume $V$ ?

Obviously, for this probability, which is a 'statistical probability', ${ }^{16}$ one obtains the value

$$
W^{r e l}=\left[\frac{V}{V_{0}}\right]^{n}
$$

from this, by applying Boltzmann's principle, one obtains

$$
S-S_{0}=k \ln \left(\frac{V}{V_{0}}\right)^{n} \text {. }
$$

By 'probability ....relative to the original' we take it Einstein means the ratio of the probabilities of the two states, so that, in accordance with (14), its logarithm is the difference in the entropies of the two states. ${ }^{17}$ And whether or not (13) really is obvious: as before, we take Einstein to mean that if independent, the probability for the joint state of all $n$ moveable points is the product of the probabilities for each; or at least that this is true of the relative probabilities. The relative probability that a single particle, initially in volume $V_{0}$, is

\footnotetext{
${ }^{15}$ Einstein (1905 p.96). To avoid confusion, we write $W^{r e l}$ where Einstein wrote $W$.

${ }^{16}$ The reference is to his preference for an ergodic interpretation of probabilities, rather than Boltzmann's combinatorial method, that he had emphasised in his early papers of 1903 and 1904.

17 This is consonant with e.g. Pais (1982 p.72); I am not aware of any alternative reading, although terminology differs (thus Norton (2016) speaks of 'transition probabilities').
} 
subsequently in the sub-volume $V$, is $V / V_{0}$; hence the relative probability that this is true of all $n$ particles is the product of the relative probabilities of each, Eq.(13).

This is, 'obviously', consistent with the volume dependence of the probability on the total phase space of the $n$ particles of the form already considered:

$$
W=V^{n} \text {. }
$$

It reproduces the required relative probability, in that

$$
W^{r e l}=\frac{W}{W_{0}}=\left(\frac{V}{V_{0}}\right)^{n} .
$$

It is the same as the ratio of probabilities for Gibbs' specific phase. But the ratio of probabilities for Gibbs' generic phases is exactly the same:

$$
\widetilde{W}^{r e l}=\frac{\widetilde{W}}{\widetilde{W}_{0}}=\left(\frac{V}{V_{0}}\right)^{n}
$$

even though $\widetilde{W}$, as given by Eq.(6), cannot be factorised. Non-interacting moveable points that are indistinguishable in Gibbs sense are not mutually independent in Einstein's sense, yet yield the same relative probability and have the same volume dependence of their entropy as do mutually independent movable points. The Wien law, concerning this kind of fluctuation, does not discriminate between them.

Would it be better to read Einstein's notion of independence as requiring the factorising only of relative probabilities? In that case indistinguishable particles in Gibbs' sense may come out as statistically independent after all. But that contravenes what we have learned from Gibbs' paradox in $\S 2$. Indeed, consider a fluctuation of a rather different sort than the one Einstein imagined, in which $n_{A}$ particles initially occupying volume $V$ are subsequently found in the sub-volume $V_{A}$, and the remaining $n_{B}$ particles, initially in volume $V$, are subsequently found in the disjoint sub-volume $V_{B}$, where $V=V_{A}+V_{B}$. Suppose first that the $n_{A}$ particles are statistically independent of the $n_{B}$ particles. Then the relative probability of the fluctuation should factorise into the product of the relative probability of each considered independently:

$$
W^{r e l}=W_{A}^{r e l} \cdot W_{B}^{r e l}=\left(\frac{V_{A}}{V}\right)^{n_{A}} \cdot\left(\frac{V_{B}}{V}\right)^{n_{B}} .
$$

It is the same, whether calculated as the ratio of the probability of the joint final state to the initial, or as the product of the ratios of final and initial states of each separately. Suppose now that the fluctuation does not change the density, so that:

$$
\frac{\left(n_{A}+n_{B}\right)}{\left(V_{A}+V_{B}\right)} \approx \frac{n_{A}}{V_{A}} \approx \frac{n_{B}}{V_{B}}
$$

The fluctuation is then the time reverse of a process of mixing of gases, as in the Gibbs set-up. It follows there is a decrease in entropy (for $V_{A}=V_{B}$, a decrease equal to $n k \ln 2$ ), which is as to be expected, given that the reverse process yields an increase in entropy.

If now we use generic phases instead, there is no such decrease, for the same reason that the time-reverse process gives no increase. The relative probability as given by the ratio of the probability of the joint final state to the probability of the joint initial state is: 


$$
\widetilde{W}^{r e l}=\frac{V_{A}^{n_{A}} / n_{A} ! \cdot V_{B}^{n_{B}} / n_{B} !}{V^{N} / n !}
$$

which, as we have already seen, is approximately unity in the Sterling approximation. It is not the product of the relative probability for a fluctuation in the state of $n_{A}$ particles, initially occupying volume $V$, by which they are found in volume $V_{A}$, with the relative probability for a fluctuation in the state of the remaining $n_{B}$ particles, from $V$ to $V_{B}$. Eq.(7) holds here just as it did for the Gibbs paradox; therefore the relative probability does not factorise.

Our earlier conclusion therefore stands: indistinguishable classical particles in Gibbs' sense are not statistically independent in Einstein's sense, not even if Einstein's factorizability condition is only required for relative probabilities. Since photons in the Wien limit have the same functional dependence on the volume and particle number as do indistinguishable classical particles, in Gibbs' sense, the result applies as much to photons. Photons are not statistically independent, in Einstein's sense, not even in the Wien regime.

\section{§4. Gibbs' generic phase as the limit of Bose-Einstein statistics}

There are of course other solutions, or purported solutions, to the Gibbs paradox, or rather paradoxes (for it has been taken to mean many different things), than the one we have considered; and there are other criticisms, or purported criticisms, of Gibbs' concept of generic phase. But there is a clear structural correspondence between statistics as made out in terms of the generic phase, vs the specific phase, and statistics following what Einstein in his 1925 called 'Bose's statistical approach', vs that for 'statistically independent molecules', following Boltzmann's. Bose's method, in the Wien limit, is the same as Gibbs', using the generic phase.

Alas, neither this structural correspondence nor the connection with Gibbs paradox was so much as mentioned by any of the key players, nor were they for some years. ${ }^{18}$ The first to consider the matter in any detail appears to have been Paul Epstein, in a contribution to the Commentary on the Scientific Writings of J. Willard Gibbs published by Yale University, Gibbs' alma mater, in 1936. Epstein highlighted the relationship between specific vs generic phase, in Gibbs' sense, and statistical independence vs Bose's method, in Einstein's; but he also maintained, as did virtually everyone following Dirac's 1926 intervention, that the absence of trajectories plays an essential role in quantum indistinguishability, which therefore has no analogue in classical statistical mechanics (whether using specific or generic phases) ${ }^{19}-$ precisely the opposite conclusion to the one advanced here.

Epstein's intervention, whatever its merits, came too late for historians of the indistinguishability concept. It came too late for Gibbs, for by then photons were a species of bosons; gibbsons were not to be. Yet the connection was there for the taking in Einstein's discussion. The key expression for the thermodynamic probability, the signature of the new statistics, had been at the heart of the quantum enigma since it first appeared in Planck's publication of 1900 (where Planck's symbol ' $h$ ' appeared for the first time as well):

\footnotetext{
${ }^{18}$ As remarked, Schrödinger (1925) came the closest, but did not mention Gibbs or the generic phase. For further discussion, see Darrigol (1991 pp.293-8), Mehra and Rechenberg (1987 pp.361-66), Saunders (2020).

19 Epstein (1936a p.557). Epstein also argued that a discrete state space was inconsistent with a continuous dynamics, in the case of generic phase, in (1936a p.564, p.527) and (1936b p.485-91).
} 


$$
\frac{(n+z-1) !}{(z-1) ! n !}
$$

It has a simple combinatorial meaning: it is the number of ways of distributing $n$ objects, among $z$ cells, where it makes no difference as to which object is assigned each cell, but only how many. It is the count of microstates, where each is defined as a specification, of each cell, of the number of objects it contains, but not of which of the $n$ objects it contains. This was wellunderstood by 1911 if not already by Boltzmann in 1877 (who was the first to write down (15), under the interpretation, the number of ways that 'energy elements', meaning integral multiples of a unit $\varepsilon$, could be distributed among $n$ objects, again without specifying of two energy elements, each with the same number of units, which is allocated to which cell). It was surely known to Gibbs, who had studied Boltzmann's early writings assiduously. But of course Gibbs had no reason to take seriously the idea that $z$ might have a well-defined physical meaning, for that was only provided by Planck's discovery in 1900, putting $\varepsilon=h v$, and as later developed in terms of the number of elementary cells to phase space of volume $h^{3}$. If Gibbs read Planck's publications in 1900 and 1901, he did not to my knowledge comment on them; he died unexpectedly in 1903.

Planck's notation did not make evident that the numbers $n$ and $z$ were tied to a range of frequency and position, denote $[s, s+\Delta s]$. Einstein's notation, twenty-five years later, did, and following Bose, generalised it from a range of frequency to a range of momentum (as well as spatial volume). Such a range Einstein called an 'elementary region' $s$ of the 1-particle state space, of the form $V_{s} \times[p, p+\Delta p]_{s} \subset \gamma$ (a coarse-graining of the one-particle state-space, indexed by $s$ ). Dividing by $h^{3}$ gave the number of elementary cells $z_{s}$. For the number of microstates, if there are $n_{s}$ particles distributed over the $z_{s}$ cells of region $s$, and if a microstate only says how many are in each cell ('Bose's method'), Einstein wrote down the expression:

$$
\frac{\left(z_{s}+n_{s}-1\right) !}{\left(z_{s}-1\right) ! n_{s} !}
$$

immediately remarking that 'it is easily recognized' that the distribution of molecules over cells is not statistically independent. We shall denote it $\tilde{Z}_{s}$, as the analog of $\widetilde{W}_{s}$, the continuous measure on the reduced phase space of the region $s$. The total probability of a macrostate is then the total number of microstates, consistent with the specification of the numbers $n_{s}$, for all the regions $s$, i.e. the product ${ }^{20}$

$$
\tilde{Z}=\prod_{s} \frac{\left(z_{s}+n_{s}-1\right) !}{\left(z_{s}-1\right) ! n_{s} !} .
$$

Einstein contrasted this expression with that following from 'the hypothesis of statistical independence of the molecules', where 'a state is defined microscopically by the fact that for each molecule one indicates in which cell it is sitting (complexion)', ${ }^{21}$ corresponding to Boltzmann's 1877 term 'complexion'. In that case the number of microstates of $n_{s}$ particles in region $s$ is:

\footnotetext{
${ }^{20}$ The probability factorises for the elementary regions, in the same way and for the same reasons, as it does for the subsystems defined by $V_{A}$ and $V_{B}$ in the Gibbs set-up (see fn.13.).

${ }^{21}$ Einstein (1925); 'each molecule' appears to be functioning as a proper name. See Saunders $(2006,2013,2016)$ for more on philosophy of language and indistinguishability.
} 


$$
z_{s}^{n_{s}},
$$

denote $Z_{s}$, and across all the regions for given occupation numbers $n_{1}, n_{2} \ldots$ it is

$$
\frac{n !}{n_{1} ! n_{2} ! \ldots \ldots} \prod_{s} z_{s}{ }^{n_{s}}
$$

where $n$ is the total number of particles, and where the pre-factor

$$
\frac{n !}{n_{1} ! n_{2} ! \ldots \ldots}
$$

is Boltzmann's famous 'permutability', as he called it. It reflected the additional multiplicity in microstates, introduced when partitioning the total number of molecules $n$ among the elementary regions, so that $n_{1}$ are in region $1, n_{2}$ in region 2 , and so on.

The different expressions (17) and (18) yield different entropy functions. Only one can be correct - and on this point Einstein thought the question was an empirical one, that 'nature must decide'. But of course, in the case of thermal radiation, nature's decision was already known, favouring the Bose-Einstein entropy and the new statistics.

And there, more or less, Einstein left the matter. He did not comment on the Wien limit; neither did Bose (and nor, in 1926, did Dirac). We should verify that it is indeed the limit of the Gibbs generic phase. The Planck distribution is:

$$
\rho=\frac{8 \pi v^{2}}{c^{3}} \frac{h v}{e^{h v / k T}-1} .
$$

It goes over to the Wien distribution (10) in the limit $h v \gg k T$. But that is the limit in which very few modes (cells) of the electromagnetic field are excited, for the minimum required is much greater than the thermal energy available (of order $k T$ ), and those that are excited are at the lowest excitation; so the occupation numbers are all 0's and 1's, with many more 0's than 1 's. If there are $n_{s}$ photons (modes with excitation 1 ), and $z_{s}$ modes, it follows that the Wien limit is the limit $z_{s} \gg n_{s}$, whereupon, to a very good approximation:

$$
\frac{\left(z_{s}+n_{s}-1\right) !}{\left(z_{s}-1\right) ! n_{s} !} \approx \frac{z_{s}^{n_{s}}}{n_{s} !} .
$$

The number of available microstates for all elementary regions $s$ for which $z_{s} \gg n_{s}$ (as determined by the temperature) is then approximately:

$$
\prod_{s ; z_{s} \gg n_{s}} \frac{z_{s}^{n_{s}}}{n_{s} !}
$$

recovering the Maxwell-Boltzmann probability Eq.(18), save for the overall factor $n$ ! (where now $n$ is the total number of excited modes in the Wien regime). In terms of the continuous phase space measure $\widetilde{W}_{s}$ for $n_{s}$ particles in region $s, \tilde{Z}_{s}$ and $\widetilde{W}_{s}$ are proportional only in the Wien regime; in contrast, using the specific phase, we have

$$
Z_{s}=\frac{1}{\tau^{n_{s}}} W_{s}
$$

for every region $s$ and numbers $n_{s}$, where $\tau$ is the unit of phase space volume $\gamma$.

Classically $\tau$ can always be chosen as sufficiently small to ensure the Wien limit is enforced 
(only the size of the elementary regions must be kept large, so as to contain many particles, so that the Sterling approximation applies). Therefore (20) always applies, and Boltzmann's combinatorial methods can be applied unchanged, replacing cells by elementary regions; the only difference is the loss of the (unwanted) factor $n$ !. In the quantum case $\tau=h^{3}$, where $h$ is Planck's constant, whereupon the number of cells $z_{S}$ cannot be taken as arbitrarily large, and the approximation (19) fails for sufficiently large $n_{s}$ (that is, at sufficiently low temperatures).

None of this was mentioned by Einstein. The correspondence between (20 and (18) should have been obvious much earlier, for example, to the Ehrenfests, at the time of writing their famous Encyclopaedia article in 1912, for they knew of the distinction between elementary regions and cells (which indeed goes back to Boltzmann in 1877). They too did not mention it. $^{22}$

Fast-forwarding to Dirac, of course the foregoing applies to the thermal equilibrium distribution of photons. In the Wien regime, the total number of microstates in each elementary region is well-approximated by (19), so photons are not statistically independent, in Einstein's sense. The exact expression for every frequency and temperature (so long as the temperature is not so high that pair-creation of electrons becomes possible) is $\tilde{Z}_{s}$, as given by (16). Indeed, $\tilde{Z}_{s}$ is the dimension of the subspace of the Hilbert space spanned by symmetrised wavefunctions in the $n_{s}$-fold tensor product of the one-particle Hilbert space $\mathcal{H}_{s}$, corresponding to the range of frequency (momentum) $[s, s+\Delta s]$ in spatial volume $V_{s}$, where $\mathcal{H}_{s}$ has dimension $z_{S}$.

\section{$\$ 5$ Locality and entanglement}

If Einstein was wrong to think that in the Wien limit light was made up of statistically independent entities, his argument surely did show that in that regime it has a discrete structure, and consists of non-interacting localised entities ${ }^{23}$ - in that nobody has been able to come up with another interpretation of the fluctuation formula (12) that says any different. He was right in another respect as well: in recommending that light quanta be thought of as movable. That might seem an obvious presupposition of his thought-experiment (how else was the fluctuation in volume supposed to happen unless the particles were 'movable'?), but it was a first step towards investing the photon, as the concept was eventually named, with different properties at different times - in short, to considering it as the sort of entity that might have different states at different times, and hence that might have a state-space at all; whereas 'energy elements', of the sort that had been discussed by Boltzmann, Planck, and Natanson, were never thought of like that. The latter were alike in all of their properties; they were hardly thought of as capable of change at all, unless it be the transfer of energy, emission and absorption, creation and annihilation. ${ }^{24}$

\footnotetext{
${ }^{22}$ They assigned $z_{s}=\omega$ in Eq.(18), with $\omega$ an arbitrary constant independent of s; whereupon the comparison of (20) with (18) is all but invisible (Ehrenfest and Ehrenfest (1912 p.27)). Ehrenfest and Kamerlingh-Onnes in their (1914) came very close to the identification, but also missed it; see my (2020) for further discussion.

${ }^{23}$ Whether the 'movable points' must really be points will depend, presumably, on the precision with which the probability of the fluctuation is defined (perhaps only in the limit $v \rightarrow \infty$ ). I refer to Dorling (1972) for discussion, already mentioned.

${ }^{24}$ The claim is alarmingly sweeping, but seems to be borne out on closer investigation: see Saunders (2020).
} 
Did it matter, historically, that light quanta were wrongly thought to be statistically independent in the Wien regime? For those in the critical period 1905-25 prepared to extend the concept of light quanta to the Rayleigh-Jeans regime, it led to the idea that correlations only came into play in that regime. This encouraged the picture of a cooperative behaviour ('the light molecule') of quanta all in the same cell, or all in the same normal mode of cavity radiation. Einstein was sympathetic to these ideas. They were also entertained, in one of his early papers, by Louis de Broglie. Einstein warmed to this theme in his second paper on gas theory in 1925 . He recommended de Broglie's newly published $\mathrm{PhD}$ thesis, as taken up, among others, by Schrödinger. In these respects the confusion was positively beneficial, for it led Schrödinger to de Broglie's concept of matter wave and to his concept of quantisation conditions as defined by solutions to wave equations; but confusion it was.

The story did not stop there, however. Einstein called the failure of statistical independence 'an initially completely puzzling mutual influence of the molecules' (ibid, p.374). He also spoke of 'spooky' action-at-a-distance, a line of inquiry that eventually led to the EPR paradox. The latter, we know, exploits entanglement. (Anti-)symmetrisation of the wave-function also involves entanglement, and surely explains the failure of statistical independence. The two, it might be thought, are at bottom the same, an argument developed at length by Don Howard (and recently endorsed by Norton). ${ }^{25}$ Yet there are important differences: as already remarked, there is a growing body of literature to show that the particular kind of entanglement required by symmetrisation is essentially trivial. Not only is it consistent with the existence of welldefined particle trajectories, it is also insufficient to violate any Bell inequality. ${ }^{26}$ It is, I suggest, just what is required, no more and no less, to ensure that factor-position, on taking the tensorproduct of one-particle Hilbert spaces, can play no role in the definition of any physical quantity. Thus the partial trace of the state, as defined by factor-position, with respect to one or more of all other particles, delivers exactly the same density matrix whatever the factorposition (a result that has led some madmen to conclude that every electron is in exactly the same state).

Then what does explain the failure of statistical independence? An alternative but still somewhat tentative answer is that microstates should not be considered as built up out of particle motions taken one at a time; that a microstate at an instant of time supervenes globally, rather than locally, on the states of individual particles. The idea that the dice thrown all at once should yield outcomes with the same probabilities, if non-interacting, as were each die thrown sequentially in time, may be the culprit; for if $n$ dice are thrown sequentially in time, each with $z$ equiprobable outcomes, there will be

$$
\frac{n !}{n_{1} ! n_{2} ! \ldots n_{z} !}
$$

different possible temporal sequences, all with the same final set of occupation numbers $n_{1}, \ldots n_{z}$, with each sequence equiprobable -- and we are back to Maxwell-Boltzmann statistics.

\footnotetext{
${ }^{25}$ Howard (1989), Norton (2006). Howard - and Norton - may well be right that the two were connected in Einstein's thinking; whether Einstein was right to connect them is the point I am contesting.

${ }^{26}$ For triviality, see Ghirardi et al (2002), Ghirardi and Marinatto (2004), Penrose (2004); for quantum trajectories, see, in addition to my (2006), Caulton (2011), Dieks and Lubberdink (2011 pp.1057-8), Saunders (2013 p.358-9, 2016 p.169-70, 2018 p.9-10); for Bell inequalities, see Caulton (2020).
} 
It is not the right metaphor.

\section{\$6 Particle trajectories and the Gibbs paradox}

There is more to learn from the failure of statistical independence apparent in the Gibbs paradox. The Gibbs paradox is properly speaking a cluster of puzzles, where the one so-far considered made direct contact with Einstein's idea of statistical independence. Here is another formulation.

If there is to be no entropy of mixing in the Gibbs set-up, there can be no new (or comparatively few) microstates available after mixing, when the partition is removed, than were not already present before. Yet it seems there are such states available. Thus Versteegh and Dieks have recently argued:27

Consider again two gas-filled chambers, each containing $n$ identical particles. Before the partition is removed the number of available states per particle is $V / 2$. After the partition has been removed, the number of available states is $V$. The reason is that after the partition's removal it has become possible for the particles to move to the other chamber. The doubling of the number of available microstates thus expresses a physical freedom that was not present before the partition was taken away. Trajectories in space-time have become possible from the particles' initial states to states in the other chamber. Particles from the left and right sides can physically exchange their states.

Their conclusion is that according to classical theory an entropy of mixing is unavoidable, and that an extensive entropy function, in classical theory, is no more than a useful fiction. More recently, Dieks has argued indistinguishability in the classical case amounts to invariance under particle relabelling, with no physical consequences: ${ }^{28}$

For example, two widely separated electrons, following distinct trajectories and labelled $a$ and $b$, still represent exactly the same physical situation after we have exchanged labels (in the sense that the electron at the first position is now called electron $b$, and vice versa). But this example also immediately demonstrates the physical insignificance of this type of indistinguishability: in spite of the exchangeability of the labels, the particles themselves can clearly be distinguished physically - we could easily tell them apart by a detection device that is sensitive to positions. So it seems that this permutability argument, and division by $n$ ! on its basis, only relates to our mode of description and does not possess physical import. This division by $n$ ! cannot possibly lead to different predictions; which makes it highly implausible that it could be relevant for a solution of the Gibbs paradox.

It appears from this that for indistinguishability of particles to have physical import it must be impossible to tell them apart: it is indistinguishability in the sense of Boltzmann, Planck, and Natanson, not Gibbs, Bose, and Einstein. But Dieks is right that division by $n$ ! relates to our 'mode of description', specifically, to the choice of state space, whether, in the classical case, it is the reduced state space $\tilde{\Gamma}$ or the specific state space $\Gamma$, and in the quantum case, whether it is the symmetrised subspace or the full tensor product of Hilbert spaces. In all cases, with the

\footnotetext{
27 Versteegh and Dieks (2011 p.742) (I have made minor changes of notation).

${ }^{28}$ Dieks (2014 p.1308).
} 
entropy given by the volume or dimensionality of the state space, it is highly plausible that this choice will be relevant to Gibbs paradox. For consider: is it true, as Versteegh and Dieks claim, that the number of available microstates doubles on removal of the partition? Figure 1 is a space-time diagram for the Gibbs set-up. In Figure 1a, the partition remains in place throughout; in Figure 1b, at time $t^{\prime}$, it is removed. New trajectories are then possible after $t^{\prime}$. But does it follow that new states are made available at $t_{2}$ ?

We may simplify further. Figure 2 is a spacetime diagram for the 2-particle Gibbs paradox. Figure 2a shows an illustrative pair of trajectories when the partition is not removed, and Figure $2 \mathrm{~b}$ shows the kind of new trajectory possible when it is, chosen to yield the same final positions

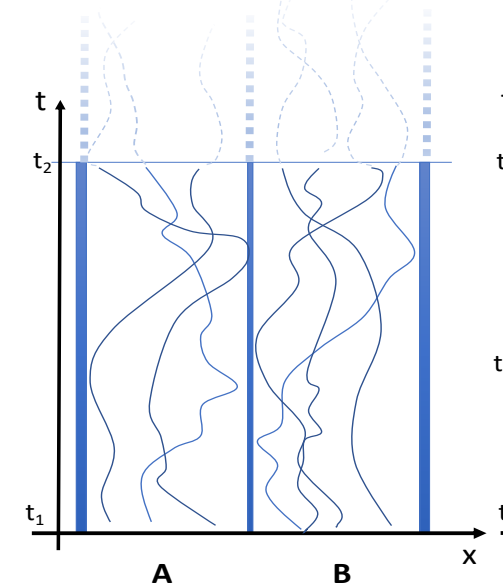

(a)

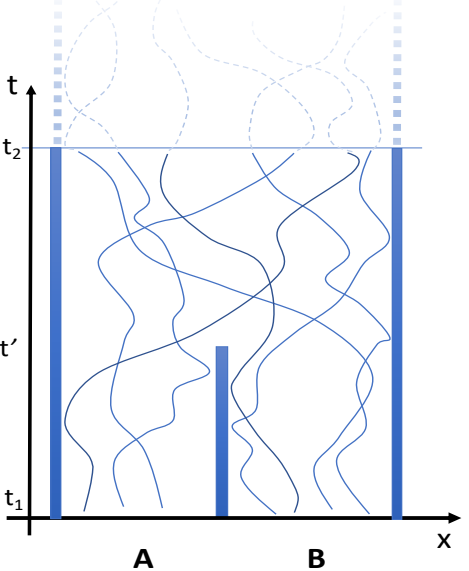

(b)

Figure 1: space-time diagram for the mixing of gases. In (a) the partition remains in place, whilst in (b) it is removed at $t^{\prime}$.

\section{]}

and momenta. The intuition to which Versteegh and Dieks appeal suggests that the two final microstates in Figure $2 \mathrm{a}$ and $2 \mathrm{~b}$ are different because they differ as to which particle has which position and momentum. This is, fairly obviously, consistent with ordinary notions of macroscopic objects, for macroscopic objects differ in their intrinsic, position- and momentumindependent properties; but for microscopic particles, whose intrinsic properties are exactly the same?

To insist that there is a difference even in this case is to insist on the use of the specific phase, in Gibbs sense. In the space of specific phases of the two particles, a continuous curve connecting two points related by particle interchange is a physical process by which each particle acquires the position and velocity of the other. In the space of specific states, it is an open curve whose end points are distinct. In the space of generic states, the initial and end points are one and the same. But the continuous curve connecting them is not obliterated - the exchange has still taken place -- it is merely that it is now a closed curve in (reduced) phase space, with beginning and end points numerically the same. (Of course these states obtain at different times; as time slices they are numerically distinct; as such each may have a different past, but that is accounted for by a change in the Hamiltonian, as time-dependent, not the state.) On using the generic phase, it remains true that new trajectories are possible, on removing the partition, but not new states; it is only that the same states (microstates) can be approached in 
new ways. ${ }^{29}$

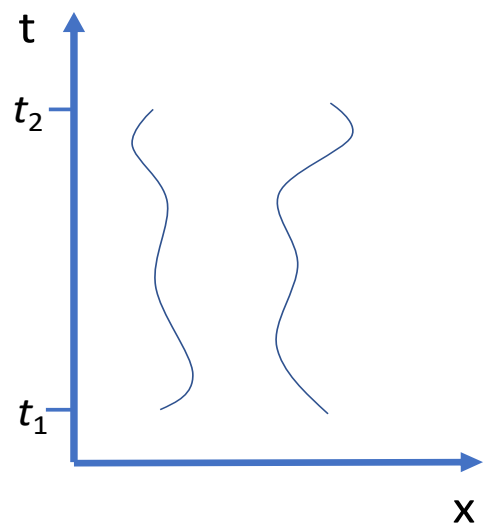

(a)

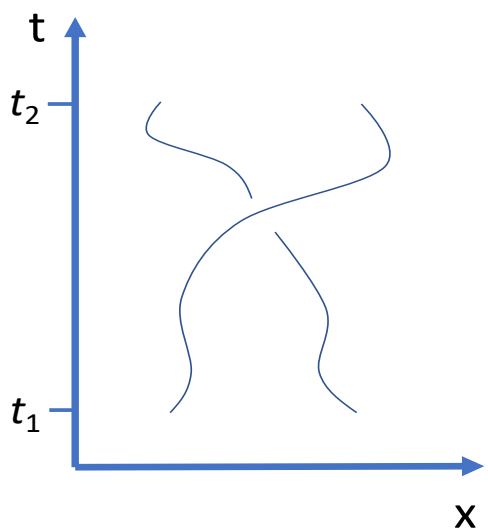

(b)

Figure 2: the 2-particle Gibbs paradox.

The Gibbs paradox, if it is a paradox, now reduces to this: are the final states of Figure 2a and $2 \mathrm{~b}$ the same? Can the state of two particles be unchanged, when each of the two particles has changed? No logical contradiction is involved. Imagine two things, each of a definite shape, say one is round and one is square; we can imagine the square slowly turning into a circle, and the circle into a square, in such a way that each occupies the same position as the other at the end.

It points, metaphysically, to the idea that the right notion of how the instantaneous state of a joint system depends on the instantaneous state of its subsystems is global. The state of the joint system supervenes on both -- it is not built up from the state of each subsystem considered individually. It may be this idea is more at home in quantum theory, and perhaps there it is forced; but it may be the right metaphysics more generally.

To see that it is forced in (conventional) quantum mechanics, consider a totally symmetrised state of two identical particles, of the form:

$$
|\Psi\rangle=\frac{1}{\sqrt{2}}(|\varphi\rangle \otimes|\psi\rangle+|\psi\rangle \otimes|\varphi\rangle)
$$

where $|\varphi\rangle \in \mathcal{H}$ is a one-particle state (and similarly $|\psi\rangle$, where $|\varphi\rangle$ and $|\psi\rangle$ are orthogonal). Let the particles be non-interacting, and suppose the unitary evolution smoothly switches the two:

$$
\widehat{U}_{t}|\varphi\rangle=|\psi\rangle ; \widehat{U}_{t}|\psi\rangle=|\varphi\rangle .
$$

Then the final state is unchanged:

\footnotetext{
${ }^{29}$ Discounting the new states in which particle numbers on each side are changed, as already mentioned.
} 


$$
\widehat{U}_{t} \otimes \widehat{U}_{t}|\Psi\rangle=|\Psi\rangle .
$$

Each of $|\varphi\rangle$ and $|\psi\rangle$ is (continuously) changed, yet the initial and final state is the same.

The same question can be posed in terms of the Feynman sum-over-paths formalism, using the same Figure 2. In computing the transition amplitude between initial and final states: do we sum the amplitudes for $2 \mathrm{a}$ and $2 \mathrm{~b}$ coherently? We should if the final state in the two cases is the same.

It was the same question posed, and answered, by Dirac in 1926, in terms of the matrix mechanics, supplemented with Schrödinger's new concept of state. He concluded:

If the positions of two of the electrons are interchanged, the new state of the atom is physically indistinguishable from the original one. In such a case one would expect only symmetrical functions of the coordinates of all the electrons to be capable of being represented by matrices. It is found that this allows one to obtain two solutions of the problem satisfying all the necessary conditions, and the theory is incapable of deciding which is the correct one. One of the solutions leads to Pauli's principle that not more than one electron can be in any given orbit, and other, when applied to the analogous problem of the ideal gas, leads to the Einstein-Bose statistical mechanics. ${ }^{30}$

Dirac explicitly considered the interchange of electrons in terms of a process connecting the original state and the interchanged state. The two are the same using Gibbs' concept of generic phase, just as they are the same using Dirac's concept of symmetrised states; and indeed, only symmetric functions of the specific phases can be represented by functions on the space of generic phases. ${ }^{31}$

There is no question that in standard quantum mechanics, for identical particles, initial and final states are identified, even if the process connecting them is a switch of the form Eq.(21). If there is a rationally compelling argument to the contrary, it awaits to be stated. But it may have seemed that the onus was on the other side to Einstein, Ehrenfest, and Lorentz, in that fateful first quarter of the last century. In my opening remarks I suggested that there may have been a genuine misconception at issue in their rejection of Gibbs' concept of generic phase. Perhaps it was this: they thought it meant, inter alia, that whether or not an interchange of like particles had actually happened was somehow a meaningless question, according to Gibbs. As realists, and given the existence of particle trajectories, they thought (rightly) that that made no sense.

The view that quantum particle indistinguishability involves the renunciation of particle trajectories has this in its favour. Departures from classical statistics involves the absence of particle trajectories, understood as well-defined orbits of 1-particle states, for those departures involve more than one particle being found in the same 1-particle state - prohibited if each has a well-defined and non-overlapping trajectory.

But in another respect the verdict is quite different. The attribution of histories to indistinguishable particles -- or more precisely, the attribution of histories, or changes of states,

\footnotetext{
${ }^{30}$ Dirac (1926 p.662). These are the two possibilities already remarked on in fn. 13 . Note that parastatistics do not arise, unless we admit microstates that differ by particle permutation alone.

${ }^{31}$ However, Dirac argued for this conclusion on the basis that the interchange is not 'physical', cementing the doctrine that indistinguishable particles cannot have trajectories. (Here he was mistaken, as (21) shows.) For further discussion see Saunders (2020).
} 
to light quanta, indeed, to an individual light quantum - the idea of a light quantum as having a state-space at all - was Bose's essential innovation in 1924. It was this that broke the conceptual deadlock over the interpretation of thermal radiation in terms of light quanta for every range of frequency and temperature, and not just in the Wien regime.

\section{References}

Bach, A. [1997] Classical Indistinguishable Particles, Berlin, Springer.

Boltzmann, L. [1877]: 'On the relationship between the second fundamental theorem of the mechanical theory of heat and probability calculations regarding the conditions for thermal equilibrium', trans. by Sharp, K. and Matschinky, F. Entropy 17 (2015) pp. 1971-2009. https://doi.org/10.3390/e17041971.

Caulton, A. [2019]: 'Entanglement by (anti-)symmetrisation does not Violate Bell's Inequalities', forthcoming.

Darrigol, O. [1986]: 'The origin of quantized matter waves', Historical Studies in the Physical and Biological Sciences, 16, pp.197-253.

- [1991]: 'Statistics and combinatorics in early quantum theory, II: Early symptoma of indistinguishability and holism', Historical Studies in the Physical and Biological Sciences, 21, pp. 237-298.

Dieks, D. [2014]: 'The logic of identity. Distinguishability and indistinguishability in classical and quantum physics.' Foundations of Physics 44, pp.1302-1316

Dieks, D. and A. Luberdink [2011]: 'How classical particles emerge from the quantum world', Foundations of Physics 41: 1051-1064. doi 10.1007/s10701-010-9515-2

Dirac, P. [1926], 'On the theory of quantum mechanics', Proceedings of the Royal Society of London A112, 661-677.

Dorling, J. [1971]: 'Einstein's introduction of photons: argument by analogy or deduction from the phenomena?', British Journal for the Philosophy of Science 22, pp. 1-8.

Ehrenfest, P. and Ehrenfest T. [1912]: The Conceptual Foundations of the Statistical Approach in Mechanics, trans. M. J. Moravcsik, New York: Dover, 1990.

Ehrenfest, P., Kammerlingh-Onnes, H. [1914]: 'Simplified deduction of the formula from the theory of combinations which Planck uses as the basis of his radiation theory', Proceedings of the Amsterdam Acadamie 17, 870-72. Reprinted in Paul Ehrenfest, Collected Scientific Papers, Klein, M. (ed).; North-Holland, 1959, pp.353-56.

Einstein A. [1905]: 'On a heuristic point of view concerning the production and transformation of light', in Stachel, J., D. Cassidy, J. Renn, and R. Schulmann (eds.) [1990]: The Collected Papers of Albert Einstein, Vol.2. The Swiss Years: Writings, 1900-1909. English Translation: trans. A. Beck, Princeton: Princeton University Press (1989).

- $\quad$ [1925]: 'Quantum theory of the ideal monatomic gas. Second part', in Buchwald, D. K., J. Illy, Z. Rosenkranz, T. Sauer, and O. Moses (eds.) [2015]: The collected papers of Albert Einstein, Vol.14. English Translation. Princeton: Princeton University Press.

Epstein, P. [1936a], 'Gibbs' methods in quantum statistics', in A Commentary on the Scientific Writings of J. Willard Gibbs VolI: Theoretical Physics, A. Haas (ed.), New Haven: Yale University Press, pp.521-84. 
- $\quad$ [1936b], 'Critical appreciation of Gibbs' statistical mechanics', in A Commentary on the Scientific Writings of J. Willard Gibbs VolI: Theoretical Physics, A. Haas (ed.), New Haven: Yale University Press, pp.461-518.

Ghirardi, G., Marinatto, L., Weber, Y. [2002]: 'Entanglement and properties of composite quantum systems: a conceptual and mathematical analysis', Journal of Statistical Physics 108, 112.

Ghirardi, G., Marinatto, L. [2004], 'General criterion for the entanglement of two indistinguishable states', Physical Review A 70, 012109.

Gibbs, J. W. [1902]: Elementary Principles in Statistical Mechanics, New York: Scribner.

Howard, D. [1990]: “Nicht sein kann was nicht sein darf” or the prehistory of EPR, 19091935: Einstein's early worries about the quantum mechanics of composite systems', in A. I. Miller (ed.) Sixty-Two Years of Uncertainty, New York: Plenum.

Mehra, J., Rechenberg, H. [1987]: The Historical Development of Quantum Theory: Vol.5, New York: Springer-Verlag.

Norton, J. D. [2006]: 'Atoms, entropy, quanta: Einstein's miraculous argument of 1905', Studies in History and Philosophy of Modern Physics 37, pp. 71-100. Available online at http://philsci-archive.pitt.edu/2477/

Pais, A. [1982]: Subtle is the Lord: the science and the life of Albert Einstein, Oxford: Oxford University Press.

Saunders, S. [2006]: 'On the explanation of quantum statistics', Studies in the History and Philosophy of Modern Physics 37, 192-11. Available online at arXiv:quant-ph/0511136

- [2013]: 'Indistinguishability', in The Oxford Handbook of Philosophy of Physics. Batterman, R. Ed.; Oxford University Press, Oxford.; pp. 340-80. http://philsciarchive.pitt.edu/12448/.

- [2016]: 'On the emergence of individuals in physics', in Individuals Across the Sciences, Guay, A., Pradeu, T. (eds), New York: Oxford University Press.

- [2018]: 'The Gibbs paradox', Entropy 20(8), 552. https://doi.org/10.3390/e20080552

- [2020], 'The concept 'indistinguishable', forthcoming in Studies in History and Philosophy of Modern Physics, https://doi.org/10.1016/j.shpsb.2020.02.003

Schrödinger, E. [1925]: 'Bemerkungen über die statistische Entropiedefinition beim idealen Gas', Preussische Akademie der Wissenschaften (Berlin), Proceeding, pp. 434-41.

Shenker, O., Hemmo, M. (eds.) [2020]: Quantum, Probabillity, Logic, Jerusalem Studies in Philosophy and History of Science. Springer, Cham. https://doi.org/10.1007/978-3-03034316-3_22

Versteegh, M., Dieks, D. [2011]: 'The Gibbs paradox and the distinguishability of identical particles', American Journal of Physics 79, 741-46. 